\title{
ON MAXIMAL ENERGY AND HOSOYA INDEX OF TREES WITHOUT PERFECT MATCHING
}

\author{
HONGBO HUA
}

\author{
(Received 10 February 2009)
}

\begin{abstract}
Let $G$ be a simple undirected graph. The energy $E(G)$ of $G$ is the sum of the absolute values of the eigenvalues of the adjacent matrix of $G$, and the Hosoya index $Z(G)$ of $G$ is the total number of matchings in $G$. A tree is called a nonconjugated tree if it contains no perfect matching. Recently, Ou ['Maximal Hosoya index and extremal acyclic molecular graphs without perfect matching', Appl. Math. Lett. 19 (2006), 652-656] determined the unique element which is maximal with respect to $Z(G)$ among the family of nonconjugated $n$-vertex trees in the case of even $n$. In this paper, we provide a counterexample to Ou's results. Then we determine the unique maximal element with respect to $E(G)$ as well as $Z(G)$ among the family of nonconjugated $n$-vertex trees for the case when $n$ is even. As corollaries, we determine the maximal element with respect to $E(G)$ as well as $Z(G)$ among the family of nonconjugated chemical trees on $n$ vertices, when $n$ is even.
\end{abstract}

2000 Mathematics subject classification: primary 05C50; secondary 05C05, 05C35.

Keywords and phrases: tree, perfect matching, energy of graph, spectra of graph, Hosoya index, $k$ matchings.

\section{Introduction}

Let $G$ be a simple graph with $n$ vertices and let $A(G)$ be its adjacency matrix. The characteristic polynomial $P_{G}(\lambda)$ of $A(G)$ is defined as

$$
P_{G}(\lambda)=\operatorname{det}(\lambda I-A(G))=\sum_{i=0}^{n} a_{i} \lambda^{n-i},
$$

where $I$ is the unit matrix of order $n$.

The roots $\lambda_{1}, \lambda_{2}, \ldots, \lambda_{n}$ of the equation $P_{G}(\lambda)=0$ are called the eigenvalues of $G$. It is evident that each $\lambda_{i}(i=1,2, \ldots, n)$ is real since $A(G)$ is symmetric.

For a graph $G$, the energy $E(G)$ of $G$ is defined to be the sum of the absolute values of the eigenvalues of the adjacent matrix of $G$.

In chemistry, the (experimentally determined) heats of formation of conjugated hydrocarbons are closely related to total $\pi$-electron energy. Within the framework

(C) 2009 Australian Mathematical Publishing Association Inc. 0004-9727/2009 \$16.00 
of the so-called HMO model the total $\pi$-electron energy is calculated from the eigenvalues of a pertinently constructed molecular graph $G$ by the equation $E(G)=$ $\sum_{i=1}^{n}\left|\lambda_{i}\right|$.

It is well known [2] that if $G$ is a bipartite graph on $n$ vertices, then $P_{G}(x)$ can be expressed as

$$
P_{G}(x)=\sum_{k=0}^{\lfloor n / 2\rfloor} a_{2 k}(G) x^{n-2 k}=\sum_{k=0}^{\lfloor n / 2\rfloor}(-1)^{k} b_{2 k}(G) x^{n-2 k},
$$

where $b_{2 k}(G) \geq 0$ for $k=0,1, \ldots,\lfloor n / 2\rfloor$. In particular, $b_{0}(G)=1$ and $b_{2}(G)$ equals the number of edges of $G$.

Suppose that $G_{1}$ and $G_{2}$ are bipartite graphs. If $b_{2 k}\left(G_{1}\right) \geq b_{2 k}\left(G_{2}\right)$ holds for all $k \geq 0$, then we write $G_{1} \succeq G_{2}$ or $G_{2} \preceq G_{1}$. If $G_{1} \succeq G_{2}$ and there exists some $k_{0}$ such that $b_{2 k_{0}}\left(G_{1}\right)>b_{2 k_{0}}\left(G_{2}\right)$, then we write $G_{1} \succ G_{2}$ or $G_{2} \prec G_{1}$. Also, we write $G_{1} \sim G_{2}$ if $G_{1} \succeq G_{2}$ and $G_{2} \preceq G_{1}$.

It is known [8] that for a bipartite graph $G$ of order $n$, its energy $E(G)$ can be expressed as the Coulson integral formula

$$
E(G)=\frac{2}{\pi} \int_{0}^{+\infty} \frac{1}{x^{2}} \ln \left(\sum_{k=0}^{\lfloor n / 2\rfloor} b_{2 k}(G) x^{2 k}\right) d x .
$$

From (1.1),

$$
\begin{aligned}
& G_{1} \succ G_{2} \Rightarrow E\left(G_{1}\right)>E\left(G_{2}\right), \\
& G_{1} \succeq G_{2} \Rightarrow E\left(G_{1}\right) \geq E\left(G_{2}\right) .
\end{aligned}
$$

The Hosoya index of $G$ is the total number of matchings in $G$, namely

$$
Z(G)=\sum_{k=0}^{\lfloor n / 2\rfloor} m(G ; k),
$$

where $n$ is the number of vertices in $G$, and $m(G ; k)$ is the number of $k$-matchings in $G$. A $k$-matching of $G$ is a $k$-element subset of its edge set, in which any two edges are mutually independent.

Another formula (see [6]) for the Hosoya index of a graph $G$ is

$$
\ln Z(G)=\sum_{+} \ln \left(1+\lambda_{j}^{2}\right)
$$

where the summation is over all positive eigenvalues of $G$. It is convenient to set $m(G ; 0)=1, m(G ; 1)=|\mathcal{E}(G)|$ and $m(G ; k)=0$ (for $k>n / 2$ ), where $|\mathcal{E}(G)|$ is the number of edges in $G$. According to Sach's theorem [2], if $G$ is a tree, then $b_{2 k}(G)=m(G ; k)$. Thus,

$$
\begin{aligned}
& G_{1} \succ G_{2} \Rightarrow Z\left(G_{1}\right)>Z\left(G_{2}\right), \\
& G_{1} \succeq G_{2} \Rightarrow Z\left(G_{1}\right) \geq Z\left(G_{2}\right) .
\end{aligned}
$$




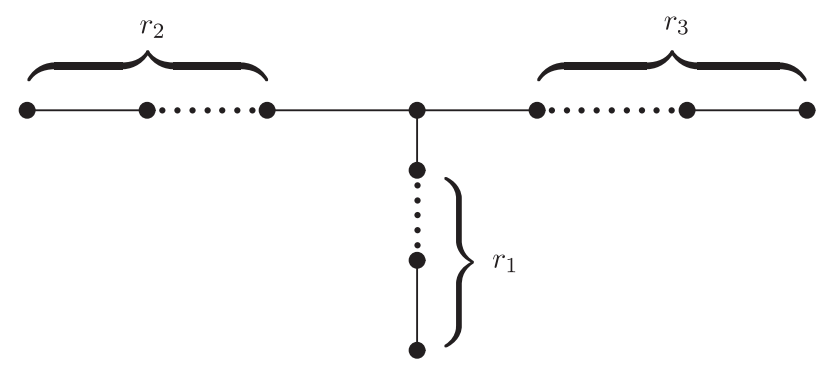

FIGURE 1. The graph $T_{r_{1}, r_{2}, r_{3}}$.

There are numerous recent results on these two subjects: see $[1,4,5,7,10,12$, $13,15,16,21-24,26]$ for graph energy, and [6, 9, 11, 14, 17, 19, 20, 23, 25] for the Hosoya index.

It is well known that among all $n$-vertex trees, the path $P_{n}$ is the unique maximal element with respect to $E(G)$ as well as $Z(G)$. A tree is called a nonconjugated tree if it contains no perfect matching. When $n$ is odd, the path $P_{n}$ is still the unique element which is maximal with respect to $E(G)$ as well as $Z(G)$ among all nonconjugated $n$-vertex trees. So it is of interest to find the maximal element with respect to $E(G)$ as well as $Z(G)$ among all nonconjugated $n$-vertex trees for the case when $n$ is even. $\mathrm{Ou}[18]$ investigated the above problem and determined the unique element which is maximal with respect to $Z(G)$. Unfortunately, Ou's results have been found to be incorrect.

In this paper, we reconsider this question and determine the unique maximal element with respect to $E(G)$ as well as $Z(G)$ among all nonconjugated $n$-vertex trees for the case when $n$ is even. As corollaries, we also determine the maximal element with respect to $E(G)$ as well as $Z(G)$ among the family of nonconjugated chemical trees on $n$ vertices when $n$ is even.

\section{Revisiting Ou's results}

Let $T_{r_{1}, r_{2}, r_{3}}$ be the star-like tree as shown in Figure 1.

If a graph $G$ contains a perfect matching, we say that $G$ has $\mathcal{P} \mathcal{M}$. Let $\mathcal{N} \mathcal{T}_{n}$ denote the set of trees of $n$ vertices, which possess no $\mathcal{P} \mathcal{M}$. Recently, Ou [18] claimed the following results.

LEMMA A. [18, Lemma 4] Let $T$ be a $4 m$-vertex tree and $k$ be a nonnegative integer. If $T \in \mathcal{N} \mathcal{T}_{4 m}$, then $m(T ; k) \leq m\left(T_{1,2 m-1,2 m-1} ; k\right)$ with equality holding if and only if $T \cong T_{1,2 m-1,2 m-1}$.

LEMMA B. [18, Lemma 5] Let $T$ be a $4 m+2$-vertex tree and $k$ be a nonnegative integer. If $T \in \mathcal{N} \mathcal{T}_{4 m+2}$, then $m(T ; k) \leq m\left(T_{1,2 m+1,2 m-1} ; k\right)$ with equality holding if and only if $T \cong T_{1,2 m+1,2 m-1}$.

Let $F_{n}$ denote the $n$th Fibonacci number. 
Theorem C. [18, Theorem 1] Let $T$ be a $4 m$-vertex tree and $k$ be a nonnegative integer. If $T \in \mathcal{N} \mathcal{T}_{4 m}$, then $Z(T) \leq 2 F_{2 m} F_{2 m+1}$ with equality holding if and only if $T \cong T_{1,2 m-1,2 m-1}$.

TheOREM D. [18, Theorem 2] Let $T$ be a $4 m+2$-vertex tree and $k$ be a nonnegative integer. If $T \in \mathcal{N} \mathcal{T}_{4 m+2}$, then $Z(T) \leq F_{2 m+2}^{2}+F_{2 m} F_{2 m+1}$ with equality holding if and only if $T \cong T_{1,2 m+1,2 m-1}$.

Lemmas A and B are evidently false, which can easily be seen from the following counterexample to Lemma A.

EXAMPLE 2.1. Let $n=12$ and consider $T_{3,3,5}$ and $T_{1,5,5}$.

From Lemma 3.1 below,

$$
\begin{aligned}
& m\left(T_{1,5,5} ; k\right)=m\left(P_{5} \cup P_{7} ; k\right)+m\left(P_{4} \cup P_{5} \cup P_{1} ; k-1\right), \\
& m\left(T_{3,3,5} ; k\right)=m\left(P_{5} \cup P_{7} ; k\right)+m\left(P_{4} \cup P_{3} \cup P_{3} ; k-1\right) .
\end{aligned}
$$

Note that $m\left(P_{3} \cup P_{3} ; 2\right)=4>3=m\left(P_{5} \cup P_{1} ; 2\right)$. So, $m\left(T_{1,5,5} ; 3\right)<m\left(T_{3,3,5} ; 3\right)$, a contradiction to $T_{3,3,5} \preceq T_{1,5,5}$, as claimed by Lemma $A$. Thus, Lemma $A$ is incorrect. Similarly, Lemma B is also incorrect, and thus Theorems C and D turn out to be incorrect.

A natural problem arising from this is the following. Among all graphs in $\mathcal{N} \mathcal{T}_{n}$ with $n$ even, which graph is the maximum element with respect to $E(G)$ as well as $Z(G)$ ? Our theorems below will provide a satisfactory answer to this question.

\section{Determining the nonconjugated tree with maximal energy and Hosoya index}

We first recall some previously established results, which will be helpful in proving our main results.

LEMMA 3.1. [8] Let $G$ be a graph with $n \geq 2$ vertices and let $u v$ be an edge in $G$. Then for all $k \geq 0$,

$$
m(G ; k)=m(G-u v ; k)+m(G-\{u, v\} ; k-1) .
$$

In particular, if $u v$ is an edge such that $v$ is a pendent vertex, then

$$
m(G ; k)=m(G-v ; k)+m(G-\{u, v\} ; k-1)
$$

for all $k \geq 0$.

LEMMA 3.2. [9] Let $P_{n}$ be a path on $n=4 s+t, 0 \leq t \leq 3$ vertices. Then

$$
\begin{aligned}
P_{n} & \succeq P_{2} \cup P_{n-2} \succeq P_{4} \cup P_{n-4} \succeq \cdots \succeq P_{2 s} \cup P_{2 s+t} \\
& \succeq P_{2 s+1} \cup P_{2 s+t-1} \succeq P_{2 s-1} \cup P_{2 s+t+1} \succeq \cdots \succeq P_{3} \cup P_{n-3} \succeq P_{1} \cup P_{n-1} .
\end{aligned}
$$

LEMMA 3.3.

(i) For $s \geq 2,3 \leq k \leq 2 s-1$ and $k$ odd, $P_{2 s+1} \cup P_{2 s-1} \succ P_{2 s+k} \cup P_{2 s-k}$.

(ii) For $s \geq 1,3 \leq k \leq 2 s+1$ and $k$ odd, $P_{2 s+1} \cup P_{2 s+1} \succ P_{2 s+k} \cup P_{2 s+2-k}$. 
PROOF. We only consider the proof of (i) here. The proof of (ii) can be derived in the same way. By Lemma 3.2, it suffices to prove that

$$
m\left(P_{2 s+1} \cup P_{2 s-1} ; 3\right)>m\left(P_{2 s+3} \cup P_{2 s-3} ; 3\right) .
$$

It is well known [3] that

$$
m\left(P_{n} ; k\right)=\left(\begin{array}{c}
n-k \\
k
\end{array}\right)
$$

and therefore

$$
\begin{aligned}
m\left(P_{2 s+1} \cup P_{2 s-1} ; 3\right)= & m\left(P_{2 s+1} ; 3\right)+m\left(P_{2 s+1} ; 2\right) m\left(P_{2 s-1} ; 1\right) \\
& +m\left(P_{2 s+1} ; 1\right) m\left(P_{2 s-1} ; 2\right)+m\left(P_{2 s-1} ; 3\right) \\
= & \left(\begin{array}{c}
2 s-2 \\
3
\end{array}\right)+\left(\begin{array}{c}
2 s-1 \\
2
\end{array}\right)\left(\begin{array}{c}
2 s-2 \\
1
\end{array}\right) \\
& \quad+\left(\begin{array}{c}
2 s \\
1
\end{array}\right)\left(\begin{array}{c}
2 s-3 \\
2
\end{array}\right)+\left(\begin{array}{c}
2 s-4 \\
3
\end{array}\right) \\
= & \frac{1}{3}\left(8 s^{3}-48 s^{2}+100 s-72\right)+8 s^{3}-18 s^{2}+20 s-2, \\
m\left(P_{2 s+3} \cup P_{2 s-3} ; 3\right)= & m\left(P_{2 s+3} ; 3\right)+m\left(P_{2 s+3} ; 2\right) m\left(P_{2 s-3} ; 1\right) \\
\quad+m\left(P_{2 s+3} ; 1\right) m\left(P_{2 s-3} ; 2\right)+m\left(P_{2 s-3} ; 3\right) & \left(\begin{array}{c}
2 s \\
3
\end{array}\right)+\left(\begin{array}{c}
2 s+1 \\
2
\end{array}\right)\left(\begin{array}{c}
2 s-4 \\
1
\end{array}\right) \\
\quad+\left(\begin{array}{c}
2 s+2 \\
1
\end{array}\right)\left(\begin{array}{c}
2 s-5 \\
2
\end{array}\right)+\left(\begin{array}{c}
2 s-6 \\
3
\end{array}\right) & \frac{1}{3}\left(8 s^{3}-48 s^{2}+148 s-168\right)+8 s^{3}-24 s^{2}+4 s+30 .
\end{aligned}
$$

It follows that

$$
m\left(P_{2 s+1} \cup P_{2 s-1} ; 3\right)-m\left(P_{2 s+3} \cup P_{2 s-3} ; 3\right)=6 s^{2}>0,
$$

which completes the proof.

PROPOSITION 3.4. Let $s(\geq 3)$ be an odd number. There exist three odd numbers $s_{1}$, $s_{2}$ and $s_{3}$ such that $s_{1}+s_{2}+s_{3}=s$ and $\left|s_{i}-s_{j}\right| \leq 2$ for $1 \leq i<j \leq 3$.

PROOF. Let $s(\geq 3)$ be an odd number. If $s=3 t$, we must have $t \equiv 1(\bmod 2)$, and thus we let $s_{i}=t$ for $1 \leq i \leq 3$. If $s=3 t+1$, we must have $t \equiv 0(\bmod 2)$, and thus we let $s_{1}=s_{2}=t+1, s_{3}=t-1$. If $s=3 t+2$, we must have $t \equiv 1(\bmod 2)$, and thus, we let $s_{1}=s_{2}=t+2, s_{3}=t$.

Denote by $\mathcal{T}_{r_{1}, r_{2}, r_{3}}$ the set of all star-like trees of the form $T_{r_{1}, r_{2}, r_{3}}$ with $r_{1}+$ $r_{2}+r_{3}+1=4 m$ or $4 m+2$, and $r_{i} \equiv 1(\bmod 2)$ for each $1 \leq i \leq 3$. Further, we let $T_{r_{1}, r_{2}, r_{3}}^{*}$ be the tree in $\mathcal{T}_{r_{1}, r_{2}, r_{3}}$ with an additional condition that $\left|r_{i}-r_{j}\right| \leq 2$ for $1 \leq i<j \leq 3$. By Proposition 3.4, $T_{r_{1}, r_{2}, r_{3}}^{*}$ is well defined. Also, such a tree is unique by Proposition 3.4. 
LEMMA 3.5. Let $T$ be any graph in $\mathcal{T}_{r_{1}, r_{2}, r_{3}}$ with $n=4 m$ or $4 m+2$, and $m \geq 1$. Then $T \preceq T_{r_{1}, r_{2}, r_{3}}^{*}$. Moreover, $T \sim T_{r_{1}, r_{2}, r_{3}}^{*}$ if and only if $T \cong T_{r_{1}, r_{2}, r_{3}}^{*}$.

PROOF. If $n=4 m$ and $m=1, T$ is isomorphic to $T_{1,1,1}$. If $n=4 m$ and $m=2, T$ is isomorphic to $T_{1,3,3}$. If $n=4 m+2$ and $m=1, T$ is isomorphic to $T_{1,1,3}$. The lemma is evidently true for these three cases. Suppose now that $T_{0}=T_{r_{1}, r_{2}, r_{3}}$ is a tree in $\mathcal{T}_{r_{1}, r_{2}, r_{3}}$ such that $T_{0} \succeq T$ for any $T \in \mathcal{T}_{r_{1}, r_{2}, r_{3}}$, but $T_{0} \nsucceq T_{r_{1}, r_{2}, r_{3}}^{*}$ both for $n=4 m, m \geq 3$ and for $n=4 m+2, m \geq 2$. Then there must exist $r_{1}$ and $r_{2}$, such that $r_{2}-r_{1} \geq 4$ (or $r_{1}-r_{2} \geq 4$ ). Assume that $r_{1}+r_{2}=2 t$. Then by Lemmas 3.1, 3.2 and 3.3, $T_{0} \prec T_{x, y, r_{3}} \in \mathcal{T}_{r_{1}, r_{2}, r_{3}}$, where $x$ and $y$ are numbers chosen by the following rules: if $t \equiv 0(\bmod 2)$, we let $x=t-1$ and $y=t+1$, or $x=t+1$ and $y=t-1$; if $t \equiv 1(\bmod 2)$, we let $x=y=t$. In fact, by Lemma 3.1,

$$
\begin{aligned}
m\left(T_{0} ; k\right) & =m\left(P_{r_{1}+r_{2}+1} \cup P_{r_{3}} ; k\right)+m\left(P_{r_{3}-1} \cup P_{r_{1}} \cup P_{r_{2}} ; k-1\right), \\
m\left(T_{x, y, r_{3}} ; k\right) & =m\left(P_{r_{1}+r_{2}+1} \cup P_{r_{3}} ; k\right)+m\left(P_{r_{3}-1} \cup P_{x} \cup P_{y} ; k-1\right) .
\end{aligned}
$$

This contradicts our choice of $T_{0}$, which completes the proof.

We mention here a well-known result, as it will play an important role in proving our main result.

Lemma 3.6. [8] Let $T$ be a tree with $n$ vertices. Then $T \preceq P_{n}$. Moreover, $T \sim P_{n}$ if and only if $T \cong P_{n}$.

In the rest of this paper, we will always denote the number of elements in a vertex subset $\mathcal{A}$ by $|\mathcal{A}|$. Before presenting our main results, it is necessary to state and prove the following lemma.

LEMMA 3.7. Let $T$ be a tree in $\mathcal{N} \mathcal{T}_{n}$ with $n=4 m$ or $n=4 m+2$, and $m \geq 1$. Then $T \preceq T_{r_{1}, r_{2}, r_{3}}^{*}$. Moreover, $T \sim T_{r_{1}, r_{2}, r_{3}}^{*}$ if and only if $T \cong T_{r_{1}, r_{2}, r_{3}}^{*}$.

PROOF. We only consider here the case when $n=4 m$. The case when $n=4 m+2$ can be dealt with in a fully analogous manner. If $m=1$, then $T_{1,1,1}$ is the unique element in $\mathcal{N} \mathcal{T}_{n}$, and the result is evidently true. So we may suppose that $m \geq 2$. Since $T \in \mathcal{N} \mathcal{T}_{n}$, then $T \nRightarrow P_{4 m}$. That is to say, $T$ has at least one vertex of degree greater than or equal to 3. Let $\Delta(T)$ be the maximum vertex-degree in $T$. Also, we use $V_{\Delta}(T)$ to denote the set $\{v \in V(T) \mid d(v)=\Delta(T)\}$. For any $T$ in $\mathcal{N} \mathcal{T}_{n}$, we clearly have $\left|V_{\Delta}(T)\right| \geq 1$. We shall prove the lemma by induction on $\left|V_{\Delta}(T)\right|$. When $\left|V_{\Delta}(T)\right|=1$, the lemma follows from Lemma 3.3 for the case $\Delta(T)=3$. So we may suppose that $\Delta(T) \geq 4$. In this case, $T$ must be isomorphic to a star-like tree with maximum vertex-degree $\Delta(T) \geq 4$. Let $d(v)=\Delta(T)$ and $T-\{v\}=P_{r_{1}} \cup P_{r_{2}} \cup \cdots \cup P_{r_{\Delta(T)}}$. It can be seen that among all the $r_{i}$, there are at least three odd positive numbers. Assume without loss of generality that $r_{i}, i=1,2,3$, are odd positive numbers. Let $Q=\left\{r_{1}, r_{2}, r_{3}, \ldots\right\}$ be the set of all odd positive numbers among $r_{1}, r_{2}, r_{3}, \ldots$, $r_{\Delta(T)}$. If there exists some $r_{i} \in Q$ such that $r_{i}=1$, then one can easily prove that $T \prec$ $T_{1,(2 m-1),(2 m-1)}$. It follows from Lemma 3.5 that $T \prec T_{1,(2 m-1),(2 m-1)} \prec T_{r_{1}, r_{2}, r_{3}}^{*}$. Suppose now that $r_{i} \geq 3$ for any $r_{i} \in Q$. Let $u$ be the vertex in $P_{r_{1}}$ (if there is more 
than one $P_{r_{1}}$ in $T-\{v\}$, we may take any one of them) such that $u$ is adjacent to $v$ in $T$. Write $T-u v=P_{r_{1}} \cup T^{\prime}$. By Lemma 3.1, we obtain

$$
\begin{aligned}
m(T ; k) & =m\left(P_{r_{1}} \cup T^{\prime} ; k\right)+m\left(P_{r_{1}-1} \cup P_{r_{2}} \cup \cdots \cup P_{\Delta_{T}(G)} ; k-1\right), \\
m\left(T_{r_{1}, x, y} ; k\right) & =m\left(P_{r_{1}} \cup P_{4 m-r_{1}} ; k\right)+m\left(P_{r_{1}-1} \cup P_{x} \cup P_{y} ; k-1\right),
\end{aligned}
$$

where $x$ and $y$ are odd numbers with the condition that $x+y=4 m-r_{1}-1$. Also, if $4 m-r_{1}-1=4 t+2$, then $x=y=2 t+1$; if $4 m-r_{1}-1=4 t$, then $x=2 t+1$ and $y=2 t-1$, or $x=2 t-1$ and $y=2 t+1$.

Note that $T^{\prime}$ is a tree of $4 m-r_{1}$ vertices not isomorphic to $P_{4 m-r_{1}}$. Then $T^{\prime} \prec$ $P_{4 m-r_{1}}$ by Lemma 3.6.

Note also that

$$
P_{r_{2}} \cup \cdots \cup P_{\Delta_{T}(G)} \prec P_{r_{2}} \cup P_{4 m-r_{1}-1-r_{2}},
$$

and that $4 m-r_{1}-1-r_{2}$ is an odd number. So

$$
P_{r_{2}} \cup \cdots \cup P_{\Delta_{T}(G)} \prec P_{r_{2}} \cup P_{4 m-r_{1}-1-r_{2}} \preceq P_{x} \cup P_{y}
$$

by Lemma 3.3. Thus, $T \prec T_{r_{1}, x, y} \preceq T_{r_{1}, r_{2}, r_{3}}^{*}$ by Lemma 3.5.

We now let $\left|V_{\Delta}(T)\right|=q \geq 2$ and suppose that the theorem is true for small values of $q$. We write $V_{p}(T)=\{v \in V(T) \mid d(v)=1\}$. For any vertex $w \in V_{\triangle}(T)$, let

$$
P_{w}(T)=\left\{u \in V_{p}(T) \mid d(u, w)<d(u, x) \text { for any } x \in V_{\triangle}(T)\right\} .
$$

It can be seen that for $\left|V_{\Delta}(T)\right| \geq 2$, there exist at least two vertices, say $x$ and $y$, in $V_{\Delta}(T)$ such that $P_{x}(T) \neq \emptyset$ and $P_{y}(T) \neq \emptyset$. Moreover, for any $z \in V_{p}(T)$, there exists a unique $w \in V_{\Delta}(T)$ such that $z \in P_{w}(T)$. Furthermore, for $\left|V_{\Delta}(T)\right| \geq 2$, there exist at least two vertices $x$ and $y$ in $V_{\Delta}(T)$ such that $\left|P_{x}(T)\right| \geq 2$ and $\left|P_{y}(T)\right| \geq 2$. Let $w$ be a vertex in $V_{\Delta}(T)$ such that $P_{w}(T)=\left\{w_{1}, w_{2}, \ldots, w_{\ell}\right\}$ for $(\ell \geq 2)$. Denote by $S_{w}$ the set of vertices (other than $w_{i}$ and $w$ ) lying on the path between $w_{i}$ and $w$ for all $i=1, \ldots, \ell$. We call the induced subtree $G\left[w, w_{1}, \ldots, w_{\ell}, S_{w}\right]$ of $T$ the pendent subtree of $T$ with respect to $w$, which is denoted by $P S_{w}(T)$. By our definition of pendent subtree and the above arguments, we know that:

- $\quad$ if $P S_{w}(T)$ is one pendent subtree of $T$, then $P S_{w}(T)$ contains no vertex other than $w$ of degree greater than or equal to 3 ;

- $\quad$ if $T$ is a tree with $\left|V_{\Delta}(T)\right| \geq 2$, then $T$ has at least two pendent subtrees.

We proceed by considering the following two cases.

CASE 1. $T$ has a pendent subtree, say $P S_{w}(T)$, which has $\mathcal{P} \mathcal{M}$.

In this case, $T$ can be viewed as the graph shown in Figure 2(a). By employing Operation I (see Figure 2) on $T$, we obtained a new graph $T^{1}$, which is obviously a graph in $\mathcal{N} \mathcal{T}_{n}$. Also, one can easily prove that $T \prec T^{1}$ by using Lemmas 3.1 and 3.6. Note that

$$
\left|V_{\triangle}\left(T^{1}\right)\right|=\left|V_{\triangle}(T)\right|-1=q-1 .
$$

Thus $T^{1} \preceq T_{r_{1}, r_{2}, r_{3}}^{*}$ by the induction assumption and then $T \prec T_{r_{1}, r_{2}, r_{3}}^{*}$. 


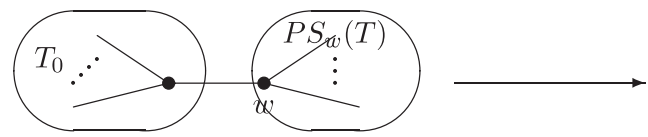

(a) $T$

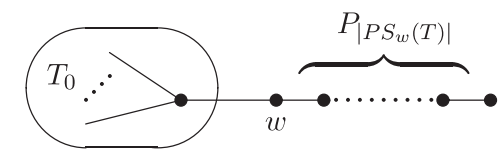

(b) $T^{1}$

Figure 2. (a) $\Rightarrow$ (b) by Operation I.

(a) $T$

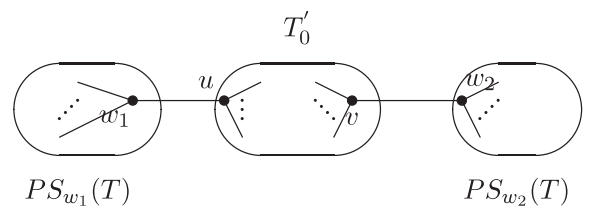

(b) $T^{2}$

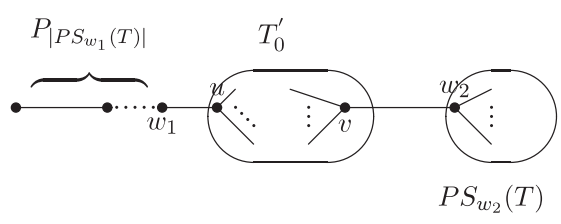

(c) $T^{3}$

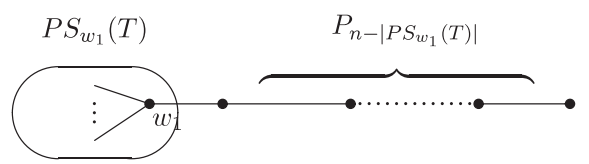

Figure 3. (a) $\Rightarrow$ (b) by Operation II; (a) $\Rightarrow$ (c) by Operation III.

CASE 2. Any pendent subtree $P S_{w}(T)$ of $T$ has no $\mathcal{P} \mathcal{M}$.

By our discussion above, if $\left|V_{\triangle}(T)\right| \geq 2$, then $T$ has at least two pendent subtrees. Suppose that the pendent subtrees of $T$ are $P S_{w_{1}}, P S_{w_{2}}, \ldots, P S_{w_{\ell}}(\ell \geq 2)$. We can always find two vertices, say $w_{1}$ and $w_{2}$, among all the $w_{i}$, such that

$$
d\left(w_{1}, w_{2}\right)=\max \left\{d\left(w_{i}, w_{j}\right) \mid 1 \leq i<j \leq l\right\} .
$$

In this case, $T$ can be viewed as the graph shown in Figure 3(a).

SubCASE 2.1. $\left|P S_{w_{1}}(T)\right| \equiv 1(\bmod 2)$ or $\left|P S_{w_{2}}(T)\right| \equiv 1(\bmod 2)$.

Assume without loss of generality that $\left|P S_{w_{1}}(T)\right| \equiv 1(\bmod 2)$.

SubCASE 2.1.1. $\left|P S_{w_{1}}(T)\right| \equiv 1(\bmod 2)$ and $G\left[V\left(T_{0}^{\prime}\right) \cup V\left(P S_{w_{2}}(T)\right) \cup\left\{w_{1}\right\}\right]$ has no $\mathcal{P} \mathcal{M}$, where $G[\bullet]=G_{T}[\bullet]$ denotes the subgraph of $T$ induced by ' $\bullet$ '.

By employing Operation II (see Figure 3) on $T$, we obtain a new graph $T^{2}$, which is obviously a graph in $\mathcal{N} \mathcal{T}_{n}$. Also, one can easily prove that $T \prec T^{2}$ by using Lemmas 3.1 and 3.6. Note that $\left|V_{\triangle}\left(T^{2}\right)\right|=\left|V_{\triangle}(T)\right|-1=q-1$. Thus $T^{2} \preceq T_{r_{1}, r_{2}, r_{3}}^{*}$ by the induction assumption and then $T \prec T_{r_{1}, r_{2}, r_{3}}^{*}$ by using Lemma 3.5.

SubCASE 2.1.2. $\left|P S_{w_{1}}(T)\right| \equiv 1(\bmod 2)$ and $G\left[V\left(T_{0}^{\prime}\right) \cup V\left(P S_{w_{2}}(T)\right) \cup\left\{w_{1}\right\}\right]$ has $\mathcal{P M}$. 
By employing Operation III (see Figure 3) on $T$, we obtain a new graph $T^{3}$, which is obviously a graph in $\mathcal{N} \mathcal{T}_{n}$. Also, one can easily prove that $T \prec T^{3}$. Note that $\left|V_{\triangle}\left(T^{3}\right)\right|=1$. Thus $T^{3} \preceq T_{r_{1}, r_{2}, r_{3}}^{*}$ by Lemma 3.5 and then $T \prec T_{r_{1}, r_{2}, r_{3}}^{*}$.

SuBCASE 2.2. $\left|P S_{w_{1}}(T)\right| \equiv 0(\bmod 2)$ and $\left|P S_{w_{2}}(T)\right| \equiv 0(\bmod 2)$.

It is obvious that $G\left[V\left(T_{0}^{\prime}\right) \cup V\left(P S_{w_{2}}(T)\right)\right]$ has no $\mathcal{P} \mathcal{M}$, since $P S_{w_{2}}(T)$ has no $\mathcal{P M}$. Thus, Operation III can be employed on $T$ once again, and we obtain the graph $T^{3}$ (see Figure 3). As in Subcase 2.1.2, we can prove our desired result.

Combining all cases completes the proof.

REMARK 3.8. According to our proof of Lemma 3.7, $P S_{w_{1}}(T)$ and $P S_{w_{2}}(T)$ are pendent subtrees chosen such that

$$
d\left(w_{1}, w_{2}\right)=\max \left\{d\left(w_{i}, w_{j}\right) \mid 1 \leq i<j \leq l\right\},
$$

among all pendent subtrees $P S_{w_{i}}(T)$ of $T$ for $1 \leq i \leq l$. In fact, one finds that the reasoning used in case 2 of the proof of Lemma 3.7 remains valid even when $d\left(w_{1}, w_{2}\right)=1$; that is, $T_{0}^{\prime}$ is an empty set. Moreover, Lemma 3.7 still follows, using the same technique, even when $u=v$ (see Figure 3).

From Lemma 3.7 we immediately obtain the following two theorems.

THEOREM 3.9. Let $T$ be a tree in $\mathcal{N} \mathcal{T}_{n}$ with $n=4 m$ or $n=4 m+2$, and $m \geq 1$. Then $Z(T) \leq Z\left(T_{r_{1}, r_{2}, r_{3}}^{*}\right)$. Moreover, $Z(T)=Z\left(T_{r_{1}, r_{2}, r_{3}}^{*}\right)$ if and only if $T \cong T_{r_{1}, r_{2}, r_{3}}^{*}$.

THEOREM 3.10. Let $T$ be a tree in $\mathcal{N} \mathcal{T}_{n}$ with $n=4 m$ or $n=4 m+2$, and $m \geq 1$. Then $E(T) \leq E\left(T_{r_{1}, r_{2}, r_{3}}^{*}\right)$. Moreover, $E(T)=E\left(T_{r_{1}, r_{2}, r_{3}}^{*}\right)$ if and only if $T \cong T_{r_{1}, r_{2}, r_{3}}^{*}$.

REMARK 3.11. Let $F_{n}$ denote the $n$th Fibonacci number. Recall that $F_{n}=F_{n-1}+$ $F_{n-2}$ with initial conditions $F_{0}=F_{1}=1$. Note that $Z\left(P_{0}\right)=1, Z\left(P_{1}\right)=1$ and $Z\left(P_{n}\right)=Z\left(P_{n-1}\right)+Z\left(P_{n-2}\right)$. Thus,

$$
Z\left(P_{n}\right)=F_{n}=\frac{\sqrt{5}}{5}\left[\left(\frac{1+\sqrt{5}}{2}\right)^{n+1}-\left(\frac{1-\sqrt{5}}{2}\right)^{n+1}\right] .
$$

So, for a specified value of $n$ in Theorem 3.9, we can compute the exact value of $Z\left(T_{r_{1}, r_{2}, r_{3}}^{*}\right)$.

A chemical tree is a tree in which no vertex has degree greater than 4 . If we denote by $\mathcal{N C \mathcal { T } _ { n }}$ the set of nonconjugated chemical trees on $n$ vertices, then by Theorems 3.9 and 3.10 we immediately have the following.

COROLlary 3.12. Let $T$ be a tree in $\mathcal{N C T} \mathcal{T}_{n}$ with $n=4 m$ or $n=4 m+2$, and $m \geq 1$. Then $Z(T) \leq Z\left(T_{r_{1}, r_{2}, r_{3}}^{*}\right)$. Moreover, $Z(T)=Z\left(T_{r_{1}, r_{2}, r_{3}}^{*}\right)$ if and only if $T \cong T_{r_{1}, r_{2}, r_{3}}^{*}$.

COROLlary 3.13. Let $T$ be a tree in $\mathcal{N C T}_{n}$ with $n=4 m$ or $n=4 m+2$, and $m \geq 1$. Then $E(T) \leq E\left(T_{r_{1}, r_{2}, r_{3}}^{*}\right)$. Moreover, $E(T)=E\left(T_{r_{1}, r_{2}, r_{3}}^{*}\right)$ if and only if $T \cong T_{r_{1}, r_{2}, r_{3}}^{*}$. 


\section{Acknowledgement}

This work was sponsored by Qing Lan Project for the key young teacher of Jiangsu province, People's Republic of China.

\section{References}

[1] G. Caporossi, D. Cvetković, I. Gutman and P. Hansen, 'Variable neighborhood search for extremal graphs. 2. Finding graphs with external energy', J. Chem. Inf. Comput. Sci. 39 (1999), 984-996.

[2] D. Cvetković, M. Doob and H. Sachs, Spectra of Graphs (Academic Press, New York, 1980).

[3] C. D. Godsil, Algebraic Combinatorics (Chapman \& Hall, London, 1993).

[4] I. Gutman, 'Acyclic systems with extremal Huckel $\pi$-electron energy', Theor. Chim. Acta. 45 (1977), 79-87.

[5] _ 'The energy of a graph: old and new results', in: Algebraic Combinatorics and Applications (Gößweinstein, 1999) (eds. A. Betten, A. Kohnert, R. Laue and A. Wassermann) (Springer, Berlin, 2001), pp. 196-211.

[6] I. Gutman and S. J. Cyvin, 'A new method for the enumeration of Kekulé structures', Chem. Phys. Lett. 136 (1987), 137-140.

[7] I. Gutman, B. Furtula and H. Hua, 'Bipartite unicyclic graphs with maximal, second-maximal and third-maximal energy', MATCH Commun. Math. Comput. Chem. 58 (2007), 75-82.

[8] I. Gutman and O. E. Polansky, Mathematical Concepts in Organic Chemistry (Springer, Berlin, 1986).

[9] I. Gutman and F. Zhang, 'On the ordering of graphs with respect to their matching numbers', Discrete Appl. Math. 15 (1986), 25-33.

[10] Y. Hou, 'Unicyclic graphs with minimal energy', J. Math. Chem. 3 (2001), 163-168.

[11] _ 'On acyclic systems with minimal Hosoya index', Discrete Appl. Math. 119 (2002), 251-257.

[12] H. Hua, 'On minimal energy of unicyclic graphs with prescribed girth and pendent vertices', MATCH Commun. Math. Comput. Chem. 57 (2007), 351-361.

[13] _ 'Bipartite unicyclic graphs with large energy', MATCH Commun. Math. Comput. Chem. 58 (2007), 57-73.

[14] _ 'Minimizing a class of unicyclic graphs by means of Hosoya index', Math. Comput. Modelling 48 (2008), 940-948.

[15] H. Hua and M. Wang, 'Unicyclic graphs with given number of pendent vertices and minimal energy', Linear Algebra Appl. 426 (2007), 478-489.

[16] X. Li, J. Zhang and L. Wang, 'On bipartite graphs with minimal energy', Discrete Appl. Math. 157(4) (2009), 869-873. doi: 10.1016/j.dam.2008.07.008.

[17] H. Liu and M. Lu, 'A unified approach to extremal cacti for different indices', MATCH Commun. Math. Comput. Chem. 58 (2007), 193-204.

[18] J. Ou, 'Maximal Hosoya index and extremal acyclic molecular graphs without perfect matching', Appl. Math. Lett. 19 (2006), 652-656.

[19] - 'On extremal unicyclic molecular graphs with prescribed girth and minimal Hosoya index', J. Math. Chem. 42 (2007), 423-432.

[20] - 'On extremal unicyclic molecular graphs with maximal Hosoya index', Discrete Appl. Math. 157 (2009), 391-397.

[21] D. Wang and H. Hua, 'Minimality considerations for graph energy over a class of graphs', Comput. Math. Appl. 56 (2008), 3181-3187.

[22] M. Wang, H. Hua and D. Wang, 'Minimal energy on a class of graphs', J. Math. Chem. 43 (2008), 1389-1402.

[23] W. Yan and L. Ye, 'On the maximal energy and the Hosoya index of a type of trees with many pendant vertices', MATCH Commun. Math. Comput. Chem. 53 (2005), 449-459.

[24] A. Yu and X. Lv, 'Minimum energy on trees with $k$ pendent vertices', Linear Algebra Appl. 418 (2007), 625-633. 
[25] _ 'The Merrifield-Simmons and Hosoya indices of trees with $k$ pendent vertices', J. Math. Chem. 41 (2007), 33-43.

[26] B. Zhou and F. Li, 'On minimal energies of trees of a prescribed diameter', J. Math. Chem. 39 (2006), 465-473.

HONGBO HUA, Department of Computing Science, Huaiyin Institute of Technology, Huaian, Jiangsu 223003, PR China

e-mail: hongbo.hua@gmail.com 